\title{
Research of VRP Model with Semi-soft Time Window Constraints
}

\author{
Lingxia Liu ${ }^{1, *}$, Xuexia Gao ${ }^{2}$ and Qiang Song ${ }^{3}$
}

\author{
${ }^{I}$ School of Software Engineering, Anyang Normal University, Anyang, 455000, China; ${ }^{2}$ School of Computer and Infor- \\ mation Engineering, Xinxiang University, Xinxiang, 453000, China, ${ }^{3}$ School of Computer and Information Engineering, \\ Anyang Institute of Technology, Anyang, 455000, China
}

\begin{abstract}
Vehicle routing problem is a kind of common problem in logistics distribution center, in order to solve the high delivery cost of VRP and time constraint problem, the VRP model with the object level and constraints by semi-soft time window is built. Aiming to the semi-soft time window constraints the optimization of the model is discussed. Finally the numerical examples prove the superiority of the semi-soft time window constraints than other types of time window.
\end{abstract}

Keywords: Object level, semi-soft time window constraints, VRP.

\section{INTRODUCTION}

In real life, in the case of distribution centers established, how to arrange the delivery route problem are gradually taken into account by people, the scholars at home and abroad makes a lot of exploration, such as Leon Cooper, in order to optimize the supply position to reduce transportation cost, summed up the transportation positioning problem [1]; R. Radharamanan and L.I. Choi introduced the tourism punishment to solve the TSP path problem [2]; K.C. Tan and L.H Lee et al. put forward using various heuristic algorithm to solve the transportation vehicles routing problem with time windows (VRPTW) [3]. Miguel Andres Figliozzi introduced modularization and hierarchical to improve transportation vehicle routing problem with hard and soft time windows [4]; Ali Gul Qureshi in soft time windows constraints used punishment cost to do microscopic simulation evaluation to build D-VRPSTW problem [5]. Chinese scholars Zhang Qian and others aiming to transportation location route problem, proposed a two-phase heuristic algorithm to address the problem of locating-ration and transport vehicle route problem [6]. Zhenggang Dan and Linning Cai used time windows to constrain transportation vehicles and route problem (that is VRPTW), they put forward a CNP method based on vehicle selection strategy to reduce the negotiation and consultation time [7]. This paper studies the vehicle allocation and transportation route selection problem, in the case of the established distribution center and customer constraining the time, the punishment cost with semi-soft time windows constraints and the total transportation cost are taken as the target system function.

\section{PROBLEME DESCRIOTION}

The goods through the logistics distribution center reaching to the customer designated location is one of important distribution way in the logistics system, on the one hand, each customer has some demand for the goods, involving to the time constraints, for example, the customers hope the transport vehicle can delivery within his specified time on time; on the other hand, the distribution center assigns the vehicles load tasks and transport routes, which will involve the cubed out problem, effective transportation and minimization of cost problem, also will be restricted by cost and route, and the high cost has been one of the focuses of logistics center. However, in the case of transport vehicles, first of all they should start from the distribution center with full of goods, according to the transport routes to complete the distribution tasks ordered by customer, and then return back to the distribution center.

The distribution center is guided by the customer demands to do ration and vehicle route arrangement, that is what goods, how many goods, and when to reach. The problem can also be described as: there is a distribution center with a known transportation vehicles number $\mathrm{M}$ (the vehicle minimum capacity is $\mathrm{L}$, and the maximum load is $\mathrm{H}$ ), the customer number is $\mathrm{N}$, the customer demand is $\mathrm{j}(\mathrm{L}<\mathrm{j}<\mathrm{H})$, there are the corresponding time window constraints to each customer demand, it is required to satisfy the customer demand with the minimum total cost of the transport vehicles distribution path.

When vehicle transport routes are arranged, the following questions should be considered:

(1) The allocation of customers. One is the classification of the customers, the customer category is different, and the strategies adopted are also corresponding different. For example the key customers with great data to and from should strengthen the maintenance to ensure the transport efficiency and service quality; the second is the partition to the customers, customers can be blocked with different distances in order to adopt different transportation strategy to deal with.

(2) The transportation cost. One is the vehicle's fuel surcharge, tolls, maintenance cost, depreciation cost; the 
second is the defray to the employees, including the employee's salary, welfare, etc.; the third is the customer's punishment cost, which refers to the extra expenses not according to the customer request to delivery on time; the fourth is other fees, such as the temporary dock fee that vehicles should pay in delivery area, urban health expenses, etc. Thus the above factors should be taken into emphasis to consider the distribution and loading problem of vehicle transport routes.

\section{THE TIME WINDOW CONSTRAINTS OF TARGET SYSTEM}

\subsection{The Target System}

For logistics distribution center, the distribution vehicle routing problem in general should achieve the following two goals:

(1) On time delivery. Logistics distribution centers serve for customers, and the service quality is reflected in time validity, now more and more customers require the punctuality (JIT), timeliness, convenience et al. services, the logistics company's top priority is how to effectively guarantee the high quality service, reasonably arrange goods to delivery to customers in time, and make the customer satisfied, it's the first goal that logistics distribution center model to consider.

(2) The cost minimization. For logistics distribution center, it certainly hopes to get more profitable returns with less investment. So how to optimize transport vehicle routing to reduce the total cost as much as possible, this is the second important goals that logistics centers need to consider.

\subsection{Time Window Constraints}

Vehicle routing problem with time window constraints (VRPTW) is divided into hard time window constraints (VRPHTW), soft time windows constraints (VRPSTW), and semi-soft time windows constraints (VRPSSTW)[8]. VRPHTW refers to in the vehicle routing problem with time windows constraints, the vehicle must arrive in accordance with customer requirements on time, it can be allowed to wait without any punishment, due to its strict limits to delay delivery, the constraint problem in real life is not too realistic [9].

The VRPSTW refers to in the vehicle routing problem, it is assumed that the vehicle can't arrive in accordance with the time stipulated in the customer requirements, it will be punished. Suppose that the time limit are respectively $E T_{i}$ and $L T_{i}$ that customers need the vehicle to arrive on, usually there are two cases: one is when the vehicle arrives earlier, it needs to wait, that is, the vehicle arrives on customer $i$ point before $E T_{i}$, the loss of opportunity cost is involved here. Another one is the vehicle arrives later, that is, the transport vehicle arrives on customer $i$ point after $L T_{i}$ point, and the service delay needs to pay a certain amount of the fine. This paper puts forward a VRP with semi-soft time windows (VRPSSTW) combining with both of the advantages, by contrast, the semi-soft time window is more closer to the reality, considering the loss of waiting and delay penalty fees of the transport vehicles, in solving the problem model the original value of cost savings can be modified and optimized.

\section{THE ESTABLISHMENT OF THE MODEL}

\subsection{Assumptions}

Based on the above analysis, the premise condition of transportation vehicle routing model is as follows:

(1) The logistics distribution center is known, and the customer distribution points are also known.

(2) The sum of customer demands should not be higher than the maximum load of transport vehicles.

(3) Each transport vehicle takes the distribution center as the starting point, along the respective distribution route delivers the goods to the customer designated locations, and then returns back to distribution center.

(4) The demand of customers is determinate.

(5) Every customer has a transport vehicle to do service, but one vehicle can serve multiple clients.

(6) The transportation vehicle should serve according to the customer time, that is, delivery within the time specified by the clients, otherwise it will be punished.

(7) The distribution path of transport vehicles should minimize the total cost as far as possible, under the premise of meeting customer requirements.

\subsection{The Establishment of Model}

Based on above analysis, according to the known conditions the following model is established for such problems:

(1) The decision variables in the model

$$
\begin{gathered}
y_{i k}= \begin{cases}1 & \text { vehicle } k \text { completes the demand of customer point } i \\
0 & \text { otherwise }\end{cases} \\
x_{i j}^{k}= \begin{cases}1 & \text { vehicle } k \text { drives from customer } i \text { to } j \\
0 & \text { otherwise }\end{cases}
\end{gathered}
$$

The parameters description in the model

$N:\left\{\mathrm{N}_{i} \mid i=1,2, \ldots, n\right\}$ represents the number of customer points;

$M:\left\{M_{k} \mid k=1,2, \ldots, m\right\}$ is the set of transport vehicles;

$H$ : the largest load of vehicle specified;

$L:$ the lower limit of vehicle capacity;

$D_{i}$ : the goods quantity demanded of customer $i$;

$c_{0}$ : the cost that vehicle drives per kilometer;

$C_{j f}, C_{j g}$ : respectively represents the lowest penalty cost that vehicle arrives earlier or later not according to the customer demands. 
$\varepsilon_{j f}, \varepsilon_{j g}$ respectively represents the cost penalty coefficients.

$d_{i j}$ : the distance between customer point $i$ and $j$.

$\delta, \varphi$ : is the target superior right coefficient of the first class and the second class respectively;

$s_{i}, u_{i}$ : respectively represents the time that vehicle $k$ arrives customer point $i$ and leaving time;

$T_{i}=u_{i}-s_{i}:$ the discharge time of customer $i$

$E T_{i}, L T_{i}$ : the time limit on the customer $i$ requirements for the arrival of the goods

$t_{i j}$ : the time spent from customer $i$ to customer $j$;

\subsection{Building the Model}

Target function:

$\operatorname{Min} Z=\delta \sum_{i} \sum_{j} \sum_{k} c_{0} d_{i j} x_{i j}^{k}+\varphi \sum_{t} v(i)$

The constraint conditions:

$$
\begin{aligned}
& \sum_{i} D_{i} y_{i k} \leq H \quad \forall k \\
& \sum_{i} D_{i} y_{i k} \geq L \quad \forall k \\
& \sum_{k} y_{i k}=1 \quad i=1, \ldots, n \\
& \sum_{i} x_{i j}^{k}-\sum_{i} x_{j i}^{k}=0 \quad j=1, \ldots, n ; \quad \forall k \\
& \sum_{i} x_{i j}^{k}=y_{j k} \quad j=1, \ldots, n ; \quad \forall k \\
& \sum_{j} x_{i j}^{k}=y_{j k} \quad i=1, \ldots, n ; \quad \forall k \\
& \sum_{i \in S} \sum_{j \in S} x_{i j}^{k} \leq|S|-1 \quad S \subseteq\{0, \ldots, n\}, 2 \leq|\mathrm{S}| \leq \mathrm{n}-1 ; \forall k \\
& x_{i j}^{k}\left(s_{j}-s_{i}\right) \geq 0 \quad \forall i, j \in N ; k \in M \\
& v(i)= \begin{cases}c_{i f}+\varepsilon_{i f}\left(2\left[E T_{j}-s_{j}\right)+1\right] / 2 & \mathrm{~s}_{j}<E T_{j} \\
0 \quad \mathrm{ET}_{j} \leq s_{j} \leq L T_{j} ; & \mathrm{j}=1, \ldots, \mathrm{n} \\
c_{j g}+\varepsilon_{j g}\left(\left[2\left(s_{j}-L T_{j}\right)+1\right] / 2\right. & \mathrm{s}_{j}>L T_{j}\end{cases} \\
& x_{i j}^{k}=0 \text { or } 1 \Rightarrow u_{i}+t_{i j} \leq s_{j} i, j=1, \ldots, n ; \forall k \\
& y_{i k}=0 \quad \text { or } 1 \quad i=1, \ldots, n ; \forall k \\
& x_{i j}^{k}=0 \text { or } 1 i, j=1, \ldots, n ; \forall k
\end{aligned}
$$

In the model, the objective function (1) represents the minimum value of the total cost (including delay penalty cost and transportation cost); expression (2) indicates the total sum of the tasks assigned to the vehicle $k$ should be equal to or less than the maximum vehicle capacity $H$; expression (3) means the total sum of the tasks assigned to the vehicle $k$ should be equal to or greater than the minimum vehicle capacity $L$; expression (4) represents every customer point must be responsible only by a distribution, each customer must be severed once; expression (5) indicates the vehicle arrives customer point $j$ and also leaves from $j$; expressions (6) and (7) represent if vehicle $k$ is assigned to a particular distribution route, there must be a closed distribution route with distribution center as the start point and end point; expression (8) represents the constraints for the branch route to void the paths that the distribution center is not taken as the start and end point; expression (9) is to ensure that the order of the vehicle driving route; expression (10) means there are time constraints for vehicle to arrive on customer, that is, there is a certain time window, if the vehicle violates, it should pay penalty cost; expression (11) represents in the same route there are existence conditions for two adjacent customers; expressions (12) and (13) guarantee the decision variables meet the constraints of rounded numbers.

\section{THE OPTIMIZATION OF MODEL}

In above model the goal priority factor is introduced, in the optimization of model the semi-soft time window is introduced to make the model more specific. Semi-soft time window is expanding the latest possible service time of vehicles, that is, $L T_{j} \rightarrow L T_{j}{ }^{\prime}$, in order to get maximum limit $L T_{j}{ }^{\prime}$, suppose the maximum delay punishment cost is equal to the amount of fees that vehicles drive to meet the demand of corresponding customer points. The $c_{e}$ is taken as a unit delay penalty cost, $R$ is used to express the time that transport vehicles from the customer $j$ point return back to the logistics distribution center, $c_{0 j}$ and $c_{j 0}$ respectively represent the use-cost for vehicles from the logistics distribution center to customer $j$ and from customer $j$ returning back to logistics distribution center, here $L T_{j}{ }^{\prime}$ is defined as:

$$
L T_{j}^{\prime}=\min \left[L T_{0}-T_{j 0}, L T_{j}+\left(c_{0 j}+c_{j 0}\right) / c_{e}\right]
$$

Hereinto, the vehicle transportation cost $c_{\mathrm{ij}}$ is made up of two costs, that is, the vehicle fixed cost of the $c_{d}\left(c_{d}=c_{0} d_{\mathrm{ij}}\right.$ ' and punishment cost $v^{\prime}(i)$, according to the above variables, $C_{\mathrm{ij}}$ can be defined as:

$$
c_{\mathrm{ij}}=\left\{\begin{array}{rr}
c_{d}+c_{j f}+\varepsilon_{j f}\left(\left[2\left(E T_{i}-s_{i}\right)+1\right] / 2\right) & \mathrm{s}_{i}<E T_{i} \\
c_{d} \quad E T_{i} \leq s_{i} \leq L T_{i}^{\prime} & \mathrm{i}=1, \ldots, \mathrm{n} \\
c_{d}+c_{j g}+\varepsilon_{j g}\left(\left[2\left(s_{i}-L T_{i}^{\prime}\right)+1\right] / 2\right. & \mathrm{s}_{\mathrm{i}}>L T^{\prime}
\end{array}\right.
$$

Now the target function can be described as: 
Table 1. Customer data.

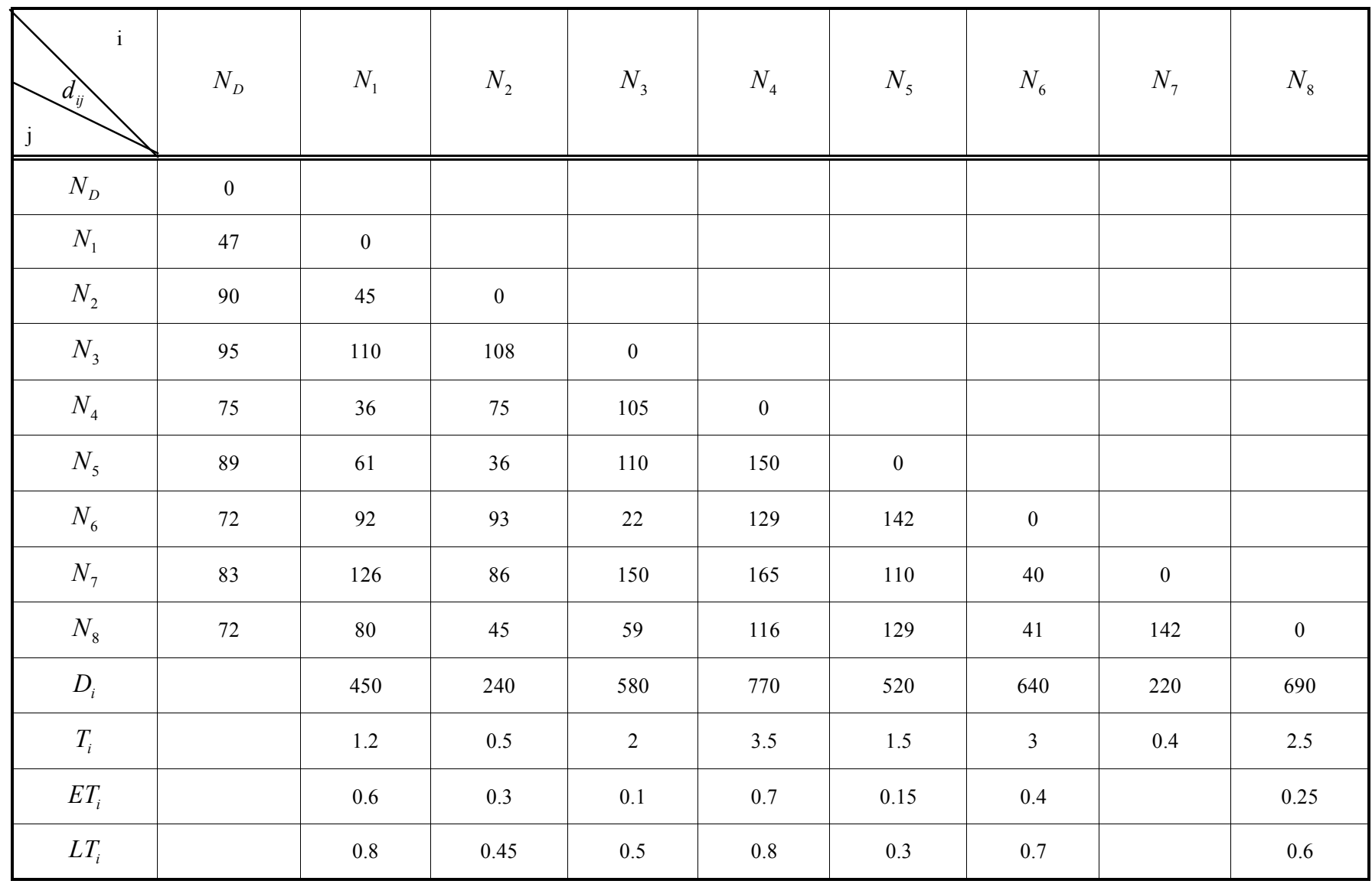

Table 2. The result analysis.

\begin{tabular}{|c|c|c|c|c|c|c|}
\hline & $\begin{array}{c}\text { Transportation } \\
\text { Cost (100 yuan) }\end{array}$ & $\begin{array}{c}\text { The Penalty Cost } \\
\text { Waiting for Delay }\end{array}$ & $\begin{array}{c}\text { Transportation } \\
\text { Plan }\end{array}$ & Cubed Out & $\begin{array}{c}\text { Iteration Times } \\
\text { Time }\end{array}$ & $\begin{array}{c}\text { Computational } \\
\text { Time }\end{array}$ \\
\hline \hline 1-VRPSTW & 710.1 & $0 ; 2.2 ; 0$ & $\begin{array}{c}\mathrm{d} 14 \mathrm{~d} ; \mathrm{d} 5267 \mathrm{~d} ; \\
\mathrm{d} 38 \mathrm{~d}\end{array}$ & $15 \% ; 54 \% ; 42.3 \%$ & $\mathrm{~min}$ & 168 \\
\hline 2-VRPSSTW & 599.0 & $0 ; 0 ; 0$ & $\mathrm{~d} 3867 \mathrm{~d} ; \mathrm{d} 5414 \mathrm{~d}$ & $71 \% ; 66 \%$ & $3 \mathrm{~min} 2 \mathrm{~s}$ \\
\hline
\end{tabular}

$\operatorname{MinZ} Z^{\prime}=\sum_{i, j \in N} \sum_{k \in M} x_{\mathrm{ij}}^{k} c_{\mathrm{ij}}$

The constraints conditions are:

$\left.s_{i}+t_{i j}-s_{j} \leq M_{\infty} 1-x_{i j}^{k}\right) \quad i, j \in N$

$E T_{j} \leq s_{j} \leq L T_{j}^{\prime} \quad j \in N$

If it needs to be further optimized, using the number of transport vehicle routing as collection points, to continue the cost optimization under the semi-soft time window constraints. Suppose $R\{r \mid r=1,2, \ldots, n\}$ is the collection of all transport vehicles distribution paths, $C_{r}$ is the total cost for vehicle to choose path, $f_{i r}$ is the times that vehicles serve for customer $i$ in the distribution path $r$, hereinto the $R$ is logarithm relationship with customer number $N$, the above problems can be further optimized, the decision variables are:

$z_{r k}=\left\{\begin{array}{rr}1 & \text { transport vehicle } k \text { selects the distribution path } r \\ 0 & \text { otherwise }\end{array}\right.$

$k \in M, r \in R$

The target function is:

$\operatorname{Minf}=\sum_{r \in R} C_{r}^{z} r k$

The constraints conditions:

$\sum_{r \in R} f_{i r} z_{r k}=1 \quad i \in N, r \in R, k \in M$

$z_{r k}=0$ or $1 \quad r \in R, k \in K$ 
Expression (20) is the minimum total cost of vehicle transportation path; expression (21) is to ensure that each customer can be served by one truck; expression (22) is used to guarantee the decision variables to meet the constraints of round numbers.

\section{EXAMPLE VERIFICATION}

There is a logistics distribution center as an example, which has three transport vehicles, the maximum capacity is $3000 \mathrm{~kg}$, the vehicle traffic fees is 100 yuan $/ \mathrm{km}$, the average speed of driving is $60 \mathrm{~km} / \mathrm{h}$, the remaining conditions are shown in Table $\mathbf{1 .}$

In VRPSSTW the unit delay $\operatorname{cost} c_{e}$ is added. From CPLEX the instance result may be calculated, which are shown in Table 2.

From table 2, to the transportation vehicle routing problem with soft time windows (1-VRPSTW), the results of numerical examples are: the transportation cost is 71010 yuan, the computation time is $5 \mathrm{~min}$, the number of iterations is 206 times; to the vehicle routing problem with semi-soft time windows (2-VRPSSTW), its computation result is 59900 yuan, computation time is $3 \mathrm{~min} 2 \mathrm{~s}$, the number of iterations is 168 times, which proves the superiority of semisoft time windows. But there are also some deficiencies, in this paper the involved cases have not enough data, the vehicle transport path problem with semi-soft time windows restriction also needs to be improved.

\section{CONCLUSION}

In order to solve the high routing cost of VRP and time constraints problem, this paper establishes a VRP model with target level and semi-soft time windows constraints, through the examples validation, the superiority of semi-soft time windows than other types of time window is proved.

\section{CONFLICT OF INTEREST}

The authors confirm that this article content has no conflict of interest.

\section{ACKNOWLEDGEMENTS}

This paper is supported by the funds of the Science and Technology Research Project of Henan province (2014), China. The project name is "The vehicle scheduling model of logistics distribution and its optimization based on the dynamic demand", and the project number is 142102210231 .

\section{REFERENCES}

[1] Leon Cooper, "The stochastic transportation-location problem", Computers \& Mathematics with Applications, vol. 4, no. 3, pp. 265 275, 1978 .

[2] Radharamanan R, Choi L I. "A branch and bound algorithm for the traveling salesman and the transportation routing problems", Computers \& Industrial Engineering, vol. 1, no. 4, pp. 236-240, 1986.

[3] Tan T C, LEE L H. "Heuristic methods for vehicle routing problem with time windows", Artificial Intelligence in Engineering, vol. 5, no.3, pp. 281-295, 2001

[4] Figliozzi Miguel Andres, "An iterative route construction and improvement algorithm for the vehicle routing problem with soft time windows", Transportation Research Part C: Emerging Technologies, vol. 18, no. 5, pp. 668-679, 2010.

[5] Qureshi Ali Gul,Taniguchi Eiichi, "A Micro simulation Based Analysis of Exact Solution of Dynamic Vehicle Routing with Soft Time Windows", Procedia-Social and Behavioral Science, vol. 39, pp. 205-216, 2012.

[6] Q. Zhang, L.Q. Gao, etc. "The two-phase heuristic algorithm to locate a transport route arrangement problem", Control and decision, vol. 12, no. 7, pp. 773-778, 2004.

[7] Z.G. Dan, L.N. Cai, "Improved Multi-agent System for the Vehicle Routing Problem with Time Windows", Tsinghua Science Technology, vol. 14, no. 3, pp. 407-412, 2009.

[8] Qureshi A G, Taniguchi E, Yamad T. "An exact solution approach for vehicle routing and scheduling problems with soft time windows", Transportation Research Part E: Logistics and Transportation Review, vol. 45, no. 6, pp. 960-977, 2006.

[9] Kallehauge Brian, Jesper Larsen, "Lagrangian duality applied to the vehicle routing problem with time windows", Computers \& Operations Research, vol. 33, no. 5, pp. 1464-1487, 2006.

Received: June 10, 2015

Revised: July 29, 2015

Accepted: August 15, 2015

(C) Liu et al.; Licensee Bentham Open.

This is an open access article licensed under the terms of the (https://creativecommons.org/licenses/by/4.0/legalcode), which permits unrestricted, noncommercial use, distribution and reproduction in any medium, provided the work is properly cited. 